\title{
A study on false alarm cancellation for spectrum usage measurements
}

\author{
Riki Mizuchii*, Kenta Umebayashi*, Janne J. Lehtomäki ${ }^{\dagger}$, and Miguel López-Benítez \\ *Graduate School of Engineering, Tokyo University of Agriculture and Technology, Japan \\ $\dagger$ Centre for Wireless Communications (CWC), University of Oulu, 90570 Oulu, Finland \\ Department of Electrical Engineering and Electronics, University of Liverpool, Liverpool L69 3GJ, U.K.
}

\begin{abstract}
Two-layer smart spectrum access (SSA) consists of spectrum sharing based on dynamic spectrum access (DSA: first layer) and spectrum awareness system (SAS: second layer). A main role of SAS is providing useful statistical information in terms of spectrum usage by long term, wide-band and widearea measurements. In this paper, we focus on signal area (SA) estimation which is a core signal processing in SAS to understand the spectrum usage. Specifically, SA estimation is used as post processing for energy detector outputs. It has been shown that Simple-SA (S-SA) estimation can enhance the spectrum usage measurement performance, but it inherently increases false alarms. For this issue, efficient false alarm cancellation technique, L-shaped false alarm cancellation (L-FC), is proposed in this paper. Numerical evaluations show that the proposed method can achieve proper detection performance while the computational cost is small compared to other methods.
\end{abstract}

Index Terms-Dynamic spectrum access, Spectrum measurement, Smart spectrum access.

\section{INTRODUCTION}

Due to fixed spectrum assignment policy and increasing demand of wireless communications, there is little room to accommodate new wireless systems. However, research by Federal Communications Commission (FCC) shows that utilization rate of spectrum in time and frequency dimensions is in range of $15 \%$ to $85 \%$ [1] and the unused spectrum is called by white space (WS). For this issue, dynamic spectrum access (DSA) [2] has been investigated. In DSA, new wireless system, such as secondary user (SU), can use spectrum owned by the existing wireless system, such as primary user (PU), if the spectrum usage by the SU will not provide any harmful interference to the PU.

In DSA, spectrum sensing and geolocation database have been investigated to find WS properly. In the case that spectrum utilization by PU is dynamic, spectrum sensing is rather appropriate since it can recognize instantaneous state of the spectrum utilization [3]-[5]. However, high sensing accuracy, low implementation cost and low latency are required in the spectrum sensing since spectrum sensing will be implemented in low cost mobile terminal and it has to protect PU properly.

One of possible approaches to achieve these requirements is using statistical information of PU's spectrum utilization such as duty cycle (DC) representing spectrum usage rate [6] in the spectrum sensing. In fact, it has been shown that the statistical information can enhance the spectrum sensing performance [7], [8]. Moreover, the statistical information can achieve more sophisticated spectrum management, channel selection and
MAC protocol design to enhance spectrum efficiency in DSA [9]-[11].

The authors have also investigated similar approach in which the statistical information is exploited as priori information and this type of DSA is denoted by smart spectrum access (SSA) [12]. One of the issues for realizing practical SAS is an implementation of measurement to obtain the priori information in an efficient way. In fact, it requires long term, wide band, and broad area spectrum usage measurement in SAS. Two-layer smart spectrum access (SSA) was proposed as a practical approach to accomplish the efficient SSA [12], [13]. It consists of two layers: the first layer is spectrum sharing based on DSA, and the second layer is spectrum awareness system which is dedicated for obtaining the priori information. The two-layer architecture can release SUs from cost in terms of implementing statistical information estimation. Since SAS has to cover broad area, a large number of spectrum sensors must be spatially deployed. It is preferable that the spectrum usage detection (detecting state of observed spectrum state either idle or occupied) process is relatively less complexity but can achieve proper detection performance.

Fourier transform based energy detector (FFT-ED) has been widely investigated in the area of wide-band spectrum sensing to observe multiple channels [14]. ED is a very simple signal detection method and it does not require any prior information of PU's signal [15]. However, detection performance of ED is not very high as compared to feature detection, and matched filter [3], [5], [16].

In this paper, we are focusing on spectrum usage measurement and a big difference compared to the spectrum sensing is latency requirement. Specifically, the spectrum sensing has to recognize instantaneous state of spectrum usage, on the other hand the spectrum usage measurement is used to estimate statistical information based on long term measurement. Therefore, a post processing for the spectrum measurement, simple-signal area (S-SA) estimation, has been proposed to enhance accuracy of the measurement in [13]. This technique exploits the fact that signal area has a rectangular shape. The outputs of Welch FFT based ED consists of two-dimensional detection result in tile which is composed of frequency bin and time slot. The detection result in each tile can be either $\mathcal{H}_{1}$ (the tile is considered to be occupied), or $\mathcal{H}_{0}$ (the tile is considered to be unoccupied). A cluster of tiles with $\mathcal{H}_{1}$ may have a rectangular shape and this cluster is denoted by 
signal area [13]. The estimation of the signal area based on the outputs can provide diversity gain and it has shown that the detection performance can be improved.

There were similar approaches as SA estimation; localization algorithm based on double-thresholding (LAD) method with adjacent cluster combining (ACC) [17], improved ED [18], and contour tracing (CT) [19]. It has been shown that SSA estimation can achieve proper accurate estimation and low computational cost, therefore we focus on S-SA estimation in this paper.

One of the significant issues of S-SA estimation is that S-SA inherently increases false alarms even though it can improve detection performance. Therefore, false alarm cancellation (FC) is added into SA estimation (FC+S-SA estimation) as a countermeasure of the increasing false alarms [13]. The FC in [13] utilizes S-SA estimations for false alarm cancellation, therefore computational cost is one of the important issues. For this issue, we propose low computational cost false alarm cancellation method which is denoted by L-shaped false alarm cancellation (L-FC). Our main contributions in this paper are summarized as follows:

- L-FC is proposed in this paper. In $\mathrm{FC}+\mathrm{S}-\mathrm{SA}$, process of FC is inserted into mid-flow of S-SA, but L-FC is independent from S-SA and the expected process is less than S-SA estimation. This structure in L+S-SA, which is combined method of L-FC and S-SA, can provide an advantage in terms of computational time.

- Numerical evaluations will show extensive comparison among L+S-SA, FC+S-SA and close-open+S-SA $(\mathrm{CO}+\mathrm{S}-$ $\mathrm{SA}$ ). Close-Open is noise (or false alarm) rejection method which is widely used in digital image processing field [20]. It will show that L+S-SA achieves the lowest computational time even if it can achieve the best detection performance compared to the others.

We summarize the major notations used throughout the paper in Table I.

TABLE I

TABLE OF NOTATIONS OF ABBREVIATIONS.

\begin{tabular}{l|l}
\hline Notation & Meaning \\
\hline S-SA & simple signal area estimation \\
L-FC & L-shaped false alarm cancellation \\
FC & false alarm cancellation \\
CO & Close-Open \\
L+S-SA & L-shaped false alarm cancellation + simple signal area \\
& estimation \\
FC+S-SA & false alarm cancellation + simple signal area estimation \\
CO+S-SA & Close-Open + simple signal area estimation \\
\hline
\end{tabular}

\section{SySTEM MODEL}

We focus on a process of spectrum usage detection in a spectrum sensor. It consists of three steps: Welch FFT based power spectrum estimation, ED, and SA estimation including FC. A role of the spectrum usage detection is to find SAs in the observing time-frequency space properly.
We assume single PU environment and bandwidth of PU signal does not change in time.

Spectrum sensors observe spectrum from $f_{c}-f_{\text {sam }} / 2$ to $f_{c}+$ $f_{\text {sam }} / 2$, where $f_{c}$ is center frequency for the observation and $f_{\text {sam }}$ is complex sampling rate. The observed signal is down converted to $f_{c}=0$ and the down converted signal is sampled with $f_{\text {sam }}$. One observation period is set to $N_{\mathrm{ob}}$ samples.

In a time slot, there are $N_{S}$ samples, which corresponds to the number of samples for single Welch FFT. Obtained samples for one observation period is divided by $N_{T S}$ time slots where $N_{T S}=N_{\mathrm{ob}} / N_{S}$.

In the Welch FFT, $N_{S}$ samples, denoted by a vector $\mathbf{r}=$ $\left(r_{0}, r_{1}, \cdots, r_{N_{S}-1}\right)^{T}$ where superscript $T$ denotes transpose, are segmented into $L$ segments of $N_{W}$ samples (referred to as segments) with $N_{O}$ overlapping samples. Overlapping ratio $N_{O} / N_{W}$ in the Welch FFT is set to 0.5 and in this case the number of segments $L$ is given by

$$
L=\frac{N_{S}-N_{W}}{N_{O}}+1=\frac{2 N_{S}}{N_{W}}-1,
$$

where $N_{S}$ and $N_{W}$ are assumed to be power of 2 for simplicity. The $i$ th segment is given by $\mathbf{r}_{\text {seg, } i}=$ $\left(r_{\left(N_{W}-N_{O}\right) \cdot i}, r_{\left(N_{W}-N_{O}\right) \cdot i+1}, \cdots, r_{\left(N_{W}-N_{O}\right) \cdot i+N_{W}-1}\right)^{T}$ where $i=$ $0,1, \cdots, L-1$.

Window function $\mathbf{w}=\left(w_{0}, w_{1}, \cdots, w_{N_{W}-1}\right)$, such as Hamming window, is applied for each segment. In this case, $N_{W}$ points FFT output for $\mathbf{r}_{\mathrm{seg}, i}$ is given by

$$
\mathbf{R}_{\mathrm{seg}, i}=\frac{1}{\sqrt{\boldsymbol{w} \boldsymbol{w}^{T}}} \mathbf{F W} \mathbf{r}_{\mathrm{seg}, i},
$$

where $\mathbf{F}=\left(e^{-j 2 \pi k l / N_{W}}\right)_{k, l=0,1, \cdots, N_{W}-1}$ is discrete Fourier transform matrix, and $\mathbf{W}$ is diagonal matrix in which diagonal elements are $\boldsymbol{w}$.

Let $R_{\text {seg, } i, n_{F}}$ denote the $n_{F}$ th element of $\mathbf{R}_{\text {seg, } i}$. Then, the $n_{F}$ th element of power spectrum vector $\mathbf{P}_{\mathrm{seg}, i}$ in the $i$ th segment is given by $\left|R_{\mathrm{seg}, i, n_{F}}\right|^{2}$. Power spectrum obtained by Welch FFT is given by

$$
\mathbf{P}=\sum_{i=0}^{L-1} \mathbf{P}_{\mathrm{seg}, i} / L
$$

Estimated power spectrum in Welch FFT is given by averaging the $L$ power spectrum, and the averaging provides the gain in ED [21]. An element of the Welch FFT output is denoted by $P_{n_{F}, n_{T}}$, where $n_{F}$ and $n_{T}$ denote index numbers of frequency bin and time slot, respectively, and $\left(n_{F}, n_{T}\right)$ denotes the coordinate of tile in the observed time-frequency space.

We assume that frequency resolution $f_{\text {sam }} / N_{W}$ and time resolutions $N_{S} / f_{\text {sam }}$ are sufficiently high, i.e., $f_{\text {sam }} / N_{W}$ is much smaller than signal bandwidth, and $N_{S} / f_{\text {sam }}$ is much smaller than continuous signal time duration. In this case, we can recognize the spectrum usage precisely.

The power spectrum obtained by Welch FFT at frequency bin $n_{F}$ and time slot $n_{T}$ is denoted by $P_{n_{F}, n_{T}}$ and $\left(n_{F}, n_{T}\right)$ denotes the coordinate of tile in the observed time-frequency space. PU's bandwidth is defined by the frequency bins when the signal power is 30 [dB] below its peak value [13], [22]. 
Output of ED in the tile $\left(n_{F}, n_{T}\right)$ is denoted by $D_{n_{F}, n_{T}}^{(\mathrm{ED})}$ and it is given by

$$
D_{n_{F}, n_{T}}^{(\mathrm{ED})}= \begin{cases}1 & \left(P_{n_{F}, n_{T}}>\eta_{\mathrm{ED}}: \mathcal{H}_{1}\right) \\ 0 & \left(\text { otherwise }: \mathcal{H}_{0}\right)\end{cases}
$$

where $\eta_{\mathrm{ED}}$ is threshold of ED. $D_{n_{F}, n_{T}}^{(\mathrm{ED})}=1$ and $D_{n_{F}, n_{T}}^{(\mathrm{ED})}=0$ indicate the estimated spectrum state in tile as $\mathcal{H}_{1}$ (PU signal is present) and $\mathcal{H}_{0}$ (PU signal is not present), respectively.

SA estimation with FC is performed to $D_{n_{F}, n_{T}}^{(\mathrm{ED})}$ in one observation period and the output of SA estimation in a tile is denoted by $D_{n_{F}, n_{T}}^{(\mathrm{SA})}$. The suffix (SA) indicates method of SA estimation with FC, such as FC+S-SA. Details of SA estimations are shown in Section III-A.

The investigated signal processing for the spectrum measurement will be performed properly even if multiple signals transmitted by different PUs are observed at the same time. However, we define signal to noise power ratio (SNR) $\gamma$ for individual transmitter which transmit signal with constant transmit power. Now we assume that $n$th complex sample $r_{n}$ is given by $r_{n}=s_{n}+z_{n}$, where $s_{n}$ represents signal component transmitted by one PU and $z_{n}$ represents the noise component. We also assume that bandwidth of the PU signal is $B, \mathbb{E}\left[s_{n}\right]=0, \mathbb{E}\left[s_{n}^{2}\right]=\sigma_{t S}^{2}$, and $z_{n} \sim \mathcal{C N}\left(0, \sigma_{t N}^{2}\right)$. Based on the assumptions, signal to noise power ratio $\gamma$ is defined by

$$
\gamma=\frac{\sigma_{f S}^{2}}{\sigma_{f N}^{2}}=\frac{\sigma_{t S}^{2} / B}{\sigma_{t N}^{2} / f_{\mathrm{sam}}},
$$

where $\sigma_{f S}^{2}=\sigma_{t S}^{2} / B$ and $\sigma_{f N}^{2}=\sigma_{t N}^{2} / f_{\text {sam }}$ are signal and noise power averaged over each bandwidth.

We define probability of detection $P_{\mathrm{D}}$ and probability of false alarm $P_{\mathrm{FA}}$ as metrics to evaluate the spectrum usage detection performance. $P_{\mathrm{D}}$ and $P_{\mathrm{FA}}$ for ED output are defined by

$$
\begin{aligned}
& P_{\mathrm{D}}^{(\mathrm{ED})}=\operatorname{Pr}\left(D_{n_{F}, n_{T}}^{(\mathrm{ED})}=1 \mid \mathcal{H}\left(n_{F}, n_{T}\right)=\mathcal{H}_{1}\right), \\
& P_{\mathrm{FA}}^{(\mathrm{ED})}=\operatorname{Pr}\left(D_{n_{F}, n_{T}}^{(\mathrm{ED})}=1 \mid \mathcal{H}\left(n_{F}, n_{T}\right)=\mathcal{H}_{0}\right),
\end{aligned}
$$

respectively, where $\mathcal{H}\left(n_{F}, n_{T}\right)$ represents the actual spectrum utilization state at tile $\left(n_{F}, n_{T}\right)$.

We also define $P_{\mathrm{D}}$ and $P_{\mathrm{FA}}$ for SA estimation output as

$$
\begin{aligned}
& P_{\mathrm{D}}^{(\mathrm{SA})}=\operatorname{Pr}\left(D_{n_{F}, n_{T}}^{(\mathrm{SA})}=1 \mid \mathcal{H}\left(n_{F}, n_{T}\right)=\mathcal{H}_{1}\right), \\
& P_{\mathrm{FA}}^{(\mathrm{SA})}=\operatorname{Pr}\left(D_{n_{F}, n_{T}}^{(\mathrm{SA})}=1 \mid \mathcal{H}\left(n_{F}, n_{T}\right)=\mathcal{H}_{0}\right),
\end{aligned}
$$

respectively.

\section{FCS FOR SA ESTIMATIONS}

\section{A. S-SA [13]}

The process of S-SA estimation consists of three steps and an example is shown in Fig. 1.

In the step 1, raster scan is performed to find a tile with $D_{n_{F}, n_{T}}^{(\mathrm{ED})}=1$ from the origin tile denoted by $\mathbf{O}$ whose coordinate is $\left(n_{F}, n_{T}\right)=(0,0)$. Direction of the raster scan is left to right and bottom to top as shown by chain line in Fig. 1.

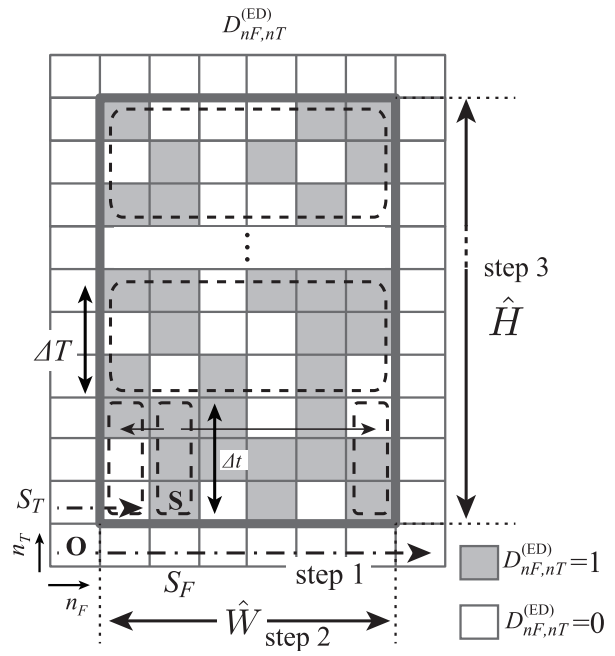

Fig. 1. S-SA estimation method. Bold line denotes genuine SA.

The tile $D_{n_{F}, n_{T}}^{(\mathrm{ED})}=1$ found by the raster scan is denoted by starting tile $\mathbf{S}$ and its coordinate is denoted by $\left(S_{F}, S_{T}\right)$.

In the step 2, we estimate the width of SA using detection mask (dashed vertical rectangle in Fig. 1). Starting from $\mathbf{S}$, the algorithm checks the detected occupancy of tiles in $n_{F}=S_{F}$ and $S_{T} \leq n_{T} \leq S_{T}+\Delta t-1$. If one or more of these tiles are determined as occupied, the algorithm moves to the right $\left(n_{F}\right.$ becomes $\left.n_{F}+1\right)$ and repeats this process. The right edge is found when all the tiles inspected are unoccupied. The left edge can be found correspondingly by moving to the opposite direction from the starting tile $\mathbf{S}$.

In the step 3, height of SA is estimated in a similar way to step 2. In this step, there are coarse height estimation and fine height estimation. Width and height of detection mask for the coarse height estimation (dashed large rectangle in Fig. 1) is set to $\hat{W}$ and $\Delta T$, respectively. This detection mask begins with its bottom row at time slot $S_{T}+\Delta t+1$. The algorithm checks the occupancy of the tiles covered by the mask with the number of $D_{n_{F}, n_{T}}^{(\mathrm{ED})}=1$ tiles and the number of $D_{n_{F}, n_{T}}^{(\mathrm{ED})}=1$ tiles is denoted by $N$. If $N \geq \eta_{C} \times \hat{W} \Delta T$, where $\eta_{C}$ is sensitivity parameter to detemine the threshold and $0<\eta_{C}<1$, the mask is moved up by $\Delta T$ time slots. This check repeats until the above condition is not satisfied and the rough height can be estimated. After this process, the fine height estimation is performed and the estimated height is denoted by $\hat{H}$. Details of S-SA estimation are shown in [13].

As confirmed in [13], S-SA has an advantage in terms of computational cost, and it can achieve relatively good detection performance. However, S-SA increases false alarms inherently. There is three main cases that increases false alarms by S-SA and the examples are shown in Fig. 2. Starting S-SA estimation from $\mathbf{S}_{\mathbf{1}}$, a few false alarms are adjacent and the process of step 2 in S-SA estimation causes additional false alarms by combining the tiles nearby the false alarms (drawn as FA1). Starting S-SA estimation from $\mathbf{S}_{\mathbf{2}}$ or $\mathbf{S}_{\mathbf{3}}$, false alarms neighbor on the left side or the bottom side of the SA and process of step 2 also causes additional false alarms (drawn 
as FA2 and FA3, respectively).

For this issue, FC was proposed in [13]. In this paper, we propose low complexity FC, i.e., L-FC. Both FC and L-FC are shown in Section III-B and Section III-C, respectively.

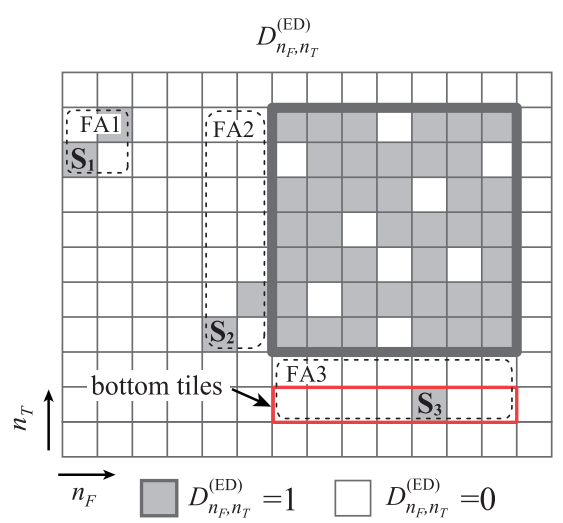

Fig. 2. False alarms caused by S-SA. Dotted rectangle shows false alarms caused by S-SA. Red rectangle shows bottom tiles of estimated SA for $\mathbf{S}_{\mathbf{3}}$.

\section{B. $F C+S-S A$}

A flow chart of $\mathrm{FC}+\mathrm{S}-\mathrm{SA}$ which corresponds to S-SA in conjunction with FC is shown in Fig. 3(a). A role of FC is re-determines the state of $\mathbf{S}$ based on two conditions and FC is performed after the step 2 in S-SA. The first condition is $\hat{W} \geq W_{t h}$, where $W_{t h}$ is threshold. This is a countermeasure for the case of FA1, specifically if the estimated width of SA is small, FC resets the state of $\mathbf{S}$ as $D_{S_{F}, S_{T}}^{(\mathrm{ED})}=0$.

The number of tiles with $D_{n_{F}, n_{T}}^{(\mathrm{ED})}=1$ at the bottom tiles of the estimated SA in the step 2 (for example, red rectangle in Fig. 2) is denoted by $N_{2}$. The second condition is $N_{2} \geq \hat{W} \times \eta_{F C}$ where $\eta_{F C}$ is sensitive parameter to determine the threshold. This is a countermeasure for the case of FA3, specifically if the number of $D_{S_{F}, S_{T}}^{(\mathrm{ED})}=1$ in the bottom tiles is small, FC resets the state of $\mathbf{S}$ as $D_{S_{F}, S_{T}}^{(\mathrm{ED})}=0$.

If the both conditions are satisfied, it goes to step 3, otherwise it goes back step 1 (raster scan).

\section{C. $L+S-S A$}

A flow chart of L+S-SA is shown in Fig. 3(b) and example of false alarm cancellation by L-FC is shown in Fig. 4. L-FC is performed right after the raster scan (step 1).

L-FC determines state of $\mathbf{S}$ based on two conditions designed by two detection masks denoted by F-mask and T-mask. The Fig. 4 shows L-FC and sizes of detection masks are $L_{f}$ tiles of F-mask in frequency domain and $L_{t}$ tiles of T-mask in time domain, respectively.

The conditions for the detection masks are as follows. In the F-mask, a detection result is $M_{L_{f}}^{(\mathrm{F})}=1$, if $x_{f} \geq \eta_{f}$ where $x_{f}$ is the number of $D_{n_{F}, n_{T}}^{(\mathrm{ED})}=1$ tiles in the detection mask, and $\eta_{f}\left(0<\eta_{f}<L_{f}\right)$ is threshold, otherwise $M_{L_{f}}^{(\mathrm{F})}=0$. In T-mask, a detection result is $M_{L_{t}}^{(\mathrm{T})}=1$, if $x_{t} \geq \eta_{t}$ where $x_{t}$ is the number of $D_{n_{F}, n_{T}}^{(\mathrm{ED})}=1$ tiles in the detection mask, and $\eta_{t}\left(0<\eta_{t}<L_{t}\right)$ is threshold, otherwise $M_{L_{t}}^{(\mathrm{T})}=0$.

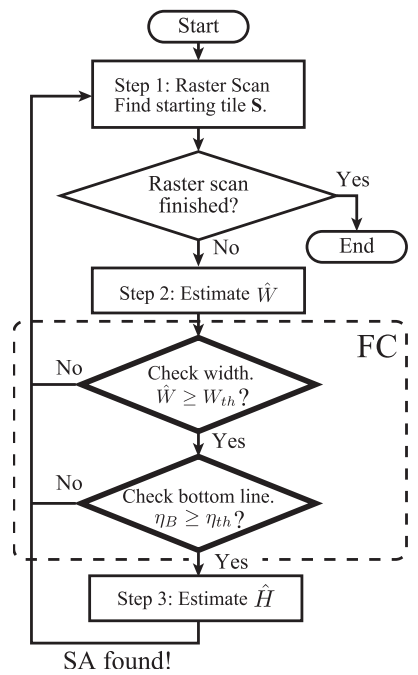

(a) $\mathrm{FC}+\mathrm{S}-\mathrm{SA}$

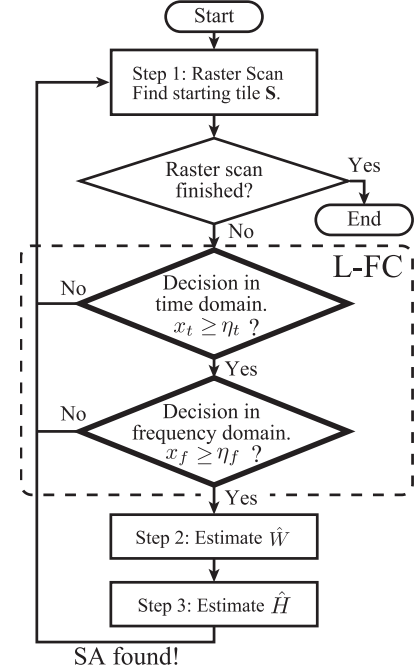

(b) L+S-SA
Fig. 3. Flow chart of S-SA methods. Thick flame is process of false alarm cancellation such as FC or L-FC.

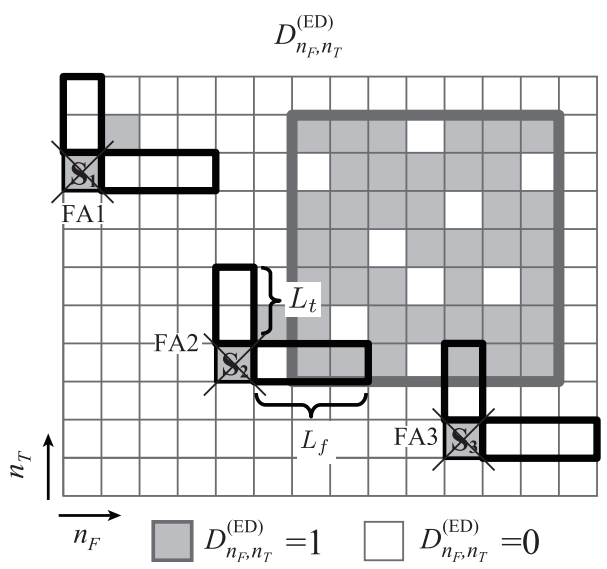

Fig. 4. Example of L-FC. Parameters are set to $L_{t}=2, \eta_{t}=1, L_{f}=3, \eta_{f}=$ 2

There are four parameters, thresholds $\eta_{f}$, and $\eta_{t}$, mask sizes, $L_{f}$, and $L_{t}$, for optimizing L-FC. We simplify the optimization problem by setting $\eta_{t}=1$ and $\eta_{f}=2$. The reason of $\eta_{f}=2$ is presence of significant correlation of $D_{n_{F}, n_{T}}^{(\mathrm{ED})}$ in frequency domain due to Hamming window, such as such as correlation coefficient between neighboring frequency bins is about 0.41 [6].

If both conditions are not satisfied, L-FC determines the state of $\mathbf{S}$ as false alarm and it goes back to the raster scan (step 1). Otherwise, steps 2 and 3 are performed.

This L-shaped detection mask can detect $\mathbf{S}$ as false alarm in the cases of FA1, FA2 and FA3 as shown in Fig. 4. This is because L-shaped detection mask check the spectrum occupancy not only in time domain (vertical direction in Fig. 4) but also frequency domain (horizontal direction in Fig. 4). On the other hand, if $\mathbf{S}$ is inside of SA and at the lower left of SA, most likely L-FC determines the state of $\mathbf{S}$ 
as signal and this is preferable for SA estimation.

In the case of L-FC, the width estimation is not necessary for $\mathrm{FC}$ and this fact leads to reduction of computational cost in L-FC compared to FC.

\section{DESIGN CRITERION}

Since we set $\eta_{t}=1$ and $\eta_{f}=2$, two parameters, $L_{t}, L_{f}$ for L-FC, have to be set properly. Design criterion for $L_{t}$ and $L_{f}$ is defined by

$$
\begin{gathered}
\left(L_{t}^{(\mathrm{opt})}, L_{f}^{(\mathrm{opt})}\right)=\underset{L_{t}, L_{f}}{\arg \max } P_{\mathrm{D}}^{(\mathrm{L}+\mathrm{S}-\mathrm{SA})}\left(L_{t}, L_{f}\right) \\
\text { s.t. } \quad P_{\mathrm{FA}}^{(\mathrm{L}+\mathrm{S}-\mathrm{SA})}\left(L_{t}, L_{f}\right)=P_{\mathrm{FA}, \text { target }}^{(\mathrm{L}+\mathrm{S}-\mathrm{SA})},
\end{gathered}
$$

where $P_{\mathrm{D}}^{(\mathrm{L}+\mathrm{S}-\mathrm{SA})}\left(L_{t}, L_{f}\right)$ and $P_{\mathrm{FA}}^{(\mathrm{L}+\mathrm{S}-\mathrm{SA})}\left(L_{t}, L_{f}\right)$ denotes $P_{\mathrm{D}}$ and $P_{\mathrm{FA}}$ of $\mathrm{L}+\mathrm{S}-\mathrm{SA}$ output, respectively and $P_{\mathrm{FA}, \text { target }}^{(\mathrm{L}+\mathrm{S}-\mathrm{SA})}$ denotes a target $P_{\mathrm{FA}}$ of $\mathrm{L}+\mathrm{S}-\mathrm{SA}$ output. In fact, analytical parameters setting is not easy. In this work, we set them based on Monte Carlo simulations since we assume spectrum usage detection does not carried out in realtime.

\section{NumERiCAl EVALUATIONS}

TABLE II

SiMULATION PARAMETERS FOR EACH SA ESTIMATION METHODS.

\begin{tabular}{l|l}
\hline Parameter & Value \\
\hline FFT size $\left(N_{S}\right)$ & $2^{10}$ \\
segment size $\left(N_{W}\right)$ & $2^{7}$ \\
overlap ratio $\left(N_{O} / N_{W}\right)$ & 0.5 \\
window function & Hamming window \\
$P_{\text {FA,target }}^{\text {(ED) }}$ & 0.01 \\
sampling rate [MHz] & 40 \\
band width of PU signal [MHz] & 20 \\
duration of one packet [us] & 256 \\
idle time [us] & 205 \\
\hline
\end{tabular}

In this section, we compare the detection performances among $\mathrm{L}+\mathrm{S}-\mathrm{SA}$ and conventional methods $(\mathrm{FC}+\mathrm{S}-\mathrm{SA}, \mathrm{CO}+\mathrm{S}-$ SA) based on constant false alarm rate (CFAR) criterion. Common simulation parameter setting is shown in Table II.

Fig. 5 shows optimum $L_{t}$ and $L_{f}$ according to (10) as a function of $\gamma$ (SNR [dB]) for different methods. The result shows that the shorter $L_{t}, L_{f}$ is better if $\gamma$ becomes higher.

\section{A. Detection Performance}

Fig. 6 shows miss detection probabilities $1-P_{\mathrm{D}}^{(\mathrm{SA})}$ as a function of $\gamma$ in terms of $\mathrm{L}+\mathrm{S}-\mathrm{SA}, \mathrm{FC}+\mathrm{S}-\mathrm{SA}, \mathrm{CO}+\mathrm{S}-\mathrm{SA}$ [20], and S-SA. In fact, the SA estimation can be interrupted as image processing, such as contour tracing. Close-open (CO) is also one of the image processing for noise rejection.

A difference between ED and S-SA show the significant gain due to $\mathrm{S}-\mathrm{SA}$ estimation. In addition, $\mathrm{FC}$ and $\mathrm{L}+\mathrm{FC}$ can also provide significant gain in the S-SA estimations. Compared to $\mathrm{CO}+\mathrm{S}-\mathrm{SA}$, since FC and L-FC are designed for the $\mathrm{SA}$ estimation, where $\mathrm{SA}$ is assumed to be a rectangle shape, FC and L-FC can achieve better detection performance in high SNR region, such as $\gamma>-3$ [dB]. In addition, we can confirm that $\mathrm{L}+\mathrm{S}-\mathrm{SA}$ can achieve the best detection performance in any $\gamma$.

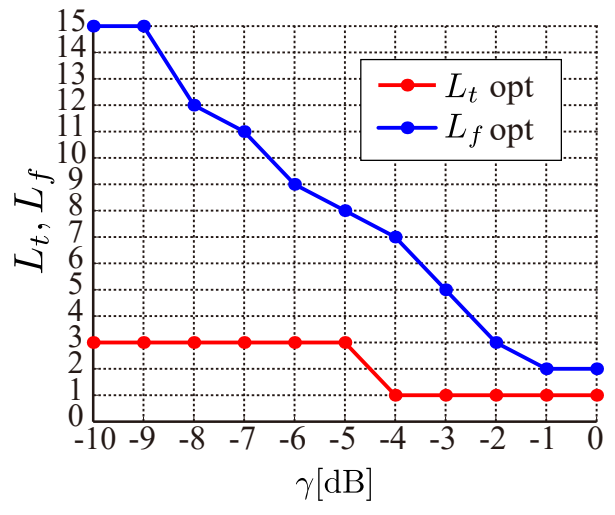

Fig. 5. Optimum parameter of $\mathrm{L}+\mathrm{S}-\mathrm{SA} . \eta_{t}$ is fixed to 1 and $\eta_{f}$ is fixed to 2. $P_{\text {FA,target }}^{(\mathrm{L}+\mathrm{S}-\mathrm{SA})}=0.01$.

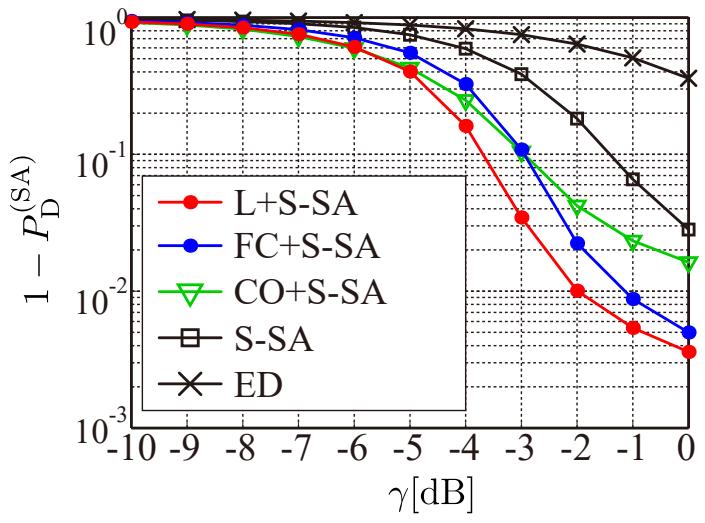

Fig. 6. Detection probability of SA estimation methods. $P_{\text {FA,target }}^{(\mathrm{SA})}=0.01$.

\section{B. Computational Time}

Fig. 7 shows computational time as a function of $P_{\mathrm{FA}}^{(\mathrm{SA})}$. In $\mathrm{CO}+\mathrm{S}-\mathrm{SA}$, false alarm detection is performed whole observation area, therefore computational time is high and does not depend on $P_{\mathrm{FA}}^{(\mathrm{SA})}$.

In $\mathrm{L}+\mathrm{S}-\mathrm{SA}$ and $\mathrm{FC}+\mathrm{S}-\mathrm{SA}$, false alarm detection is performed on $\mathbf{S}$. Therefore, in the region where $P_{\mathrm{FA}}^{(\mathrm{SA})}>0.004$, the computational time increases as $P_{\mathrm{FA}}^{(\mathrm{SA})}$ increases. Since FC requires more computational time than L-FC due to the step 2 (the width estimation), we can confirm L-FC can achieve less computational time. Obviously S-SA can achieve smallest computational time since any false alarm cancellation is not used.

In Fig. 7, computational time of $\mathrm{L}+\mathrm{S}-\mathrm{SA}$ and $\mathrm{FC}+\mathrm{S}-\mathrm{SA}$ is minimum around $P_{\mathrm{FA}}^{(\mathrm{SA})}=0.004$. The reason of this behavior is confirmed in Fig. 8 which shows $N_{\text {noise }}$ and $N_{\text {signal }}$ where $N_{\text {noise }}$ denotes the number of $\mathbf{S}$ tiles detected as false alarm by FC or L-FC, and $N_{\text {signal }}$ denotes the number of $\mathbf{S}$ tiles detected as signal by $\mathrm{FC}$ or L-FC.

In fact the behavior of computational time of $\mathrm{FC}+\mathrm{S}-\mathrm{SA}$ and behavior of $N_{\text {noise }}$ of $\mathrm{FC}+\mathrm{S}-\mathrm{SA}$. In the region where $P_{\text {FA }}^{(\mathrm{SA})}<0.004$, false alarm probability is low, and it also leads to low $P_{\mathrm{D}}^{(\mathrm{ED})}$. Therefore, one signal area may divided 
into several small portions and finally FC may detect it as false alarms. Then, computational time and $N_{\text {noise }}$ are high in the region where $P_{\mathrm{FA}}^{(\mathrm{SA})}<0.004$.

In fact, $N_{\text {noise }}$ of L-FC is also high in the region where $P_{\mathrm{FA}}^{(\mathrm{SA})}<0.004$ due to the same reason. However, computational time for L-FC is inherently low, therefore, it would not affect very much in the case of $\mathrm{L}+\mathrm{S}-\mathrm{SA}$ estimation.

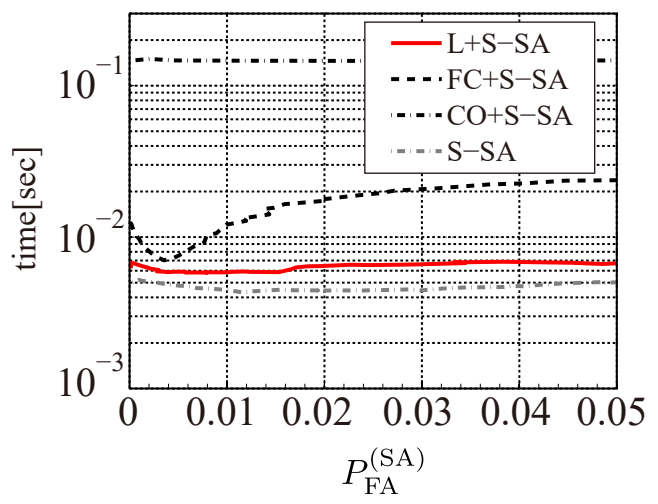

Fig. 7. Computational time of SA estimation methods. $(\gamma=-5[\mathrm{~dB}])$

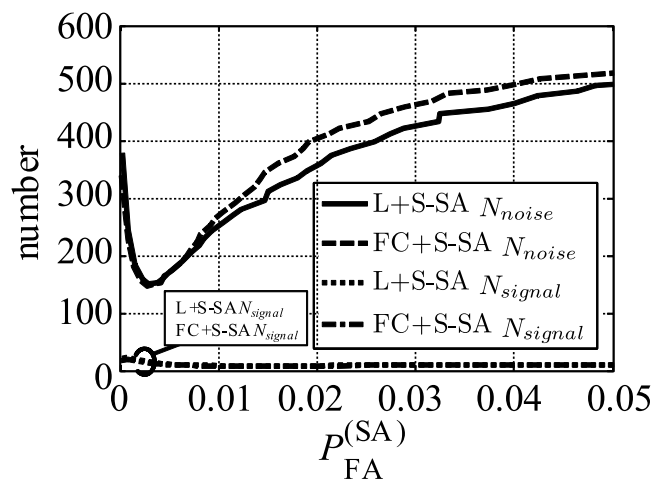

Fig. 8. Number of signal tiles $N_{\text {signal }}$ and number of noise tiles $N_{\text {noise }}$ over one observation period. $(\gamma=-5[\mathrm{~dB}])$

\section{CONCLUSION}

In this paper, we investigated the false alarm cancellation method for S-SA estimation which is a post processing of ED for efficient spectrum usage detection. We proposed L-FC which can achieve accurate detection performance and low computational time than conventional methods. L-FC has two parameters and they have to be set properly. We also show parameters setting criterion and the Monte Carlo simulation shows the proper parameters.

In numerical evaluations, we confirmed that L-FC and SSA can provide significant gain in spectrum usage detection compared to the other approaches, such as ED. In addition, we confirmed that L+S-SA with the optimum parameters can achieve the best detection performance. Moreover, L+SSA can achieve less computational time than other methods, FC+S-SA, and CO+S-SA. Finally, based on the above outcomes, it can be concluded that L+S-SA is efficient post processing of ED for the spectrum usage detection.

\section{ACKNOWLEDGMENT}

This research and development work was supported by the MIC/SCOPE \#165003006, and JSPS KAKENHI Grant Numbers JP15K06053, JP15KK0200.

\section{REFERENCES}

[1] FCC, "Spectrum Policy Task Force ET Dcket No. 02-135," Nov. 2002.

[2] I. F. Akyildiz, W. Y. Lee, M. C. Vuran, and S. Mohanty, "NeXt generation/dynamic spectrum access/cognitive radio wireless networks: A survey," Computer Networks, vol. 50, no. 13, pp. 2127-2159, Sep. 2006.

[3] L. Lu, X. Zhou, U. Onunkwo, and G. Li, "Ten years of research in spectrum sensing and sharing in cognitive radio," EURASIP J. Wireless Commun. and Net., vol. 28, Jan. 2012.

[4] J. Lunden, V. Koivunen, and H. Poor, "Spectrum Exploration and Exploitation for Cognitive Radio," IEEE Signal Process. Mag., vol. 32, no. 3, pp. 123-140, Apr. 2015.

[5] T. Yucek and H. Arslan, "A survey of spectrum sensing algorithms for cognitive radio applications," IEEE Commun. Surveys Tuts., vol. 11, no. 1, pp. 116-130, Mar. 2009.

[6] J. J. Lehtomäki, R. Vuohtoniemi, and K. Umebayashi, "On the Measurement of Duty Cycle and Channel Occupancy Rate," IEEE J. Sel. Areas Commun., vol. 31, no. 11, pp. 2555-2565, Nov. 2013.

[7] N. Wang, Y. Gao, and X. Zhang, "Adaptive spectrum sensing algorithm under different primary user utilizations," IEEE Commun. Lett., vol. 17, no. 9, pp. 1838-1841, Aug. 2013.

[8] T. Nguyen, B. L. Mark, and Y. Ephraim, "Spectrum sensing using a hidden bivariate markov model," IEEE Trans. Wireless Commun., vol. 12, no. 9, pp. 4582-4591, Aug. 2013.

[9] J. Vartiainen, M. Höyhtyä, J. Lehtomäki, and T. Bräysy, "Priority channel selection based on detection history database," in Proc. CROWNCOM, Cannes, France, Jun. 2010, pp. 1 - 5.

[10] K. Umebayashi and Y. Suzuki, "Dynamic selection of CWmin in cognitive radio networks for protecting IEEE 802.11 primary users," in Proc. CROWNCOM, Yokohama, Japan, Jun. 2011, pp. $266-270$.

[11] Y. Xu, A. Anpalagan, Q. Wu, L. Shen, Z. Gao, and J. Wang, "DecisionTheoretic Distributed Channel Selection for Opportunistic Spectrum Access: Strategies, Challenges and Solutions," IEEE Commun. Surveys Tuts., vol. 15, no. 4, pp. 1689-1713, Jan. 2013.

[12] K. Umebayashi, S. Tiiro, and J. Lehtomäki, "Development of a measurement system for spectrum awareness," in Proc. 5GU, Levi, Finland, Nov. 2014.

[13] K. Umebayashi, K. Moriwaki, R. Mizuchi, H. Iwata, S. Tiiro, J. J. Lehtomäki, M. López-benítez, and Y. Suzuki, "Simple Primary User Signal Area Estimation for Spectrum Measurement," IEICE Trans. Commun., vol. E99-B, no. 02, Feb. 2016.

[14] Q. Zhang, A. B. Kokkeler, and G. J. Smit, "An efficient multi-resolution spectrum sensing method for cognitive radio," in Proc. CHINACOM, Hangzhou, China, Aug. 2008, pp. 1226-1229.

[15] H. Urkowitz, "Energy detection of unknown deterministic signals," Proc. IEEE, vol. 55, no. 4, Apr. 1967.

[16] H. O. Kpojime and G. A. Safdar, "Interference Mitigation in CognitiveRadio-Based Femtocells," IEEE Commun. Surveys Tuts., vol. 17, no. 3, pp. 1511-1534, Jan. 2015.

[17] J. Vartiainen, H. Sarvanko, J. Lehtomäki, M. Juntti, and M. Latvaaho, "Spectrum Sensing with LAD-Based Methods," in Proc. PIMRC, Athens, Greece, Sep. 2007, pp. 1-5.

[18] M. López-benítez and F. Casadevall, "Improved energy detection spectrum sensing for cognitive radio," IET Communications, vol. 6, no. 8, p. 785, May 2012.

[19] J. Kokkoniemi and J. Lehtomaeki, "Spectrum Occupancy Measurements and Analysis Methods on the $2.45 \mathrm{GHz}$ ISM Band," in Proc. CROWNCOM, Stockholm, Sweden, Jun. 2012.

[20] P. Soille, Morphological Image Analysis. Springer Berlin Heidelberg, 2004.

[21] H. Iwata, K. Umebayashi, S. Tiiro, Y. Suzuki, and J. J. Lehtomäki, "Optimum welch FFT segment size for duty cycle estimation in spectrum awareness system," in Proc. WCNCW, New Orleans, USA, Mar. 2015, pp. 229-234.

[22] ITU-R, "SPECTRA AND BANDWIDTH OF EMISSIONS," RECOMMENDATION ITU-R SM.328-10, Dec. 1999. 